\title{
MIRRORSAR: A FRACTIONATED SPACE RADAR FOR BISTATIC, MULTISTATIC AND HIGH-RESOLUTION WIDE-SWATH SAR IMAGING
}

\author{
Gerhard Krieger, Mariantonietta Zonno, Marc Rodriguez-Cassola, Paco Lopez-Dekker", Josef Mittermayer, \\ Marwan Younis, Sigurd Huber, Michelangelo Villano, Felipe Queiroz de Almeida, Pau Prats-Iraola, Alberto Moreira
}

German Aerospace Center (DLR), Microwaves and Radar Institute, Germany

\#Delft University of Technology, The Netherlands

\begin{abstract}
This paper introduces the concept of a fractionated MirrorSAR which is based on a set of mutually separated transmitter and receiver satellites. As opposed to previously published bi- and multistatic SAR systems, the receiver satellites are considerably simplified, as their main functionality is reduced to a kind of microwave mirror (or space transponder) which routes the radar echoes towards the transmitter. The forwarded radar signals are then coherently demodulated within the transmitter by using the same oscillator that had been used for radar pulse generation. This avoids the necessity of a bidirectional phase synchronization link as currently employed in TanDEM-X. Since the needs for fully equipped radar receivers, on-board memory and downlink are also overcome, the weight and costs of the receiver satellites can be significantly reduced. This allows for a scaling of their number without cost explosion, thereby paving the way for novel applications like multibaseline SAR interferometry and single-pass tomography. Several additional opportunities make the MirrorSAR concept even more attractive. First, the separation of the transmitter and receiver front-ends reduces not only RF losses by avoiding switches and circulators, but it may also lower the peak power in the transmitter satellite by employing a frequency-modulated continuous wave (FMCW) illumination. This simplifies the design of the high-power amplifier and increases its efficiency. Second, the opportunity for continuous radar data collection enables new modes for the imaging of ultra-wide swaths with very high resolution, thereby overcoming an inherent limitation of conventional monostatic SAR systems. Third, the joint availability of all receiver signals in a centralized node offers new opportunities for efficient data compression, as the multistatic radar signals from close satellite formations are characterized by a high degree of mutual redundancy. Fourth, the use of a sufficiently separated transmitter satellite can avoid the risk for mutual illumination, which challenges the design and operation of fully-active multistatic SAR systems. Further advantages arise from the scalability and reconfigurability, which support new redundancy concepts and pave at the same time the way to new modes like MIMO-SAR tomography.
\end{abstract}

\section{INTRODUCTION}

Synthetic aperture radar (SAR) is a well-established remote sensing technique that can provide high-resolution images independent of weather and sunlight illumination. SAR images of the Earth surface are now regularly acquired by an increasing number of satellites and form the basis for a wide range of services and applications [1]. Further opportunities arise from bistatic SAR systems [2]. A prominent example is TanDEM-X, which enabled not only the acquisition of a global digital elevation model (DEM) with unprecedented accuracy and resolution, but also the demonstration of new bistatic imaging modes and techniques such as along-track

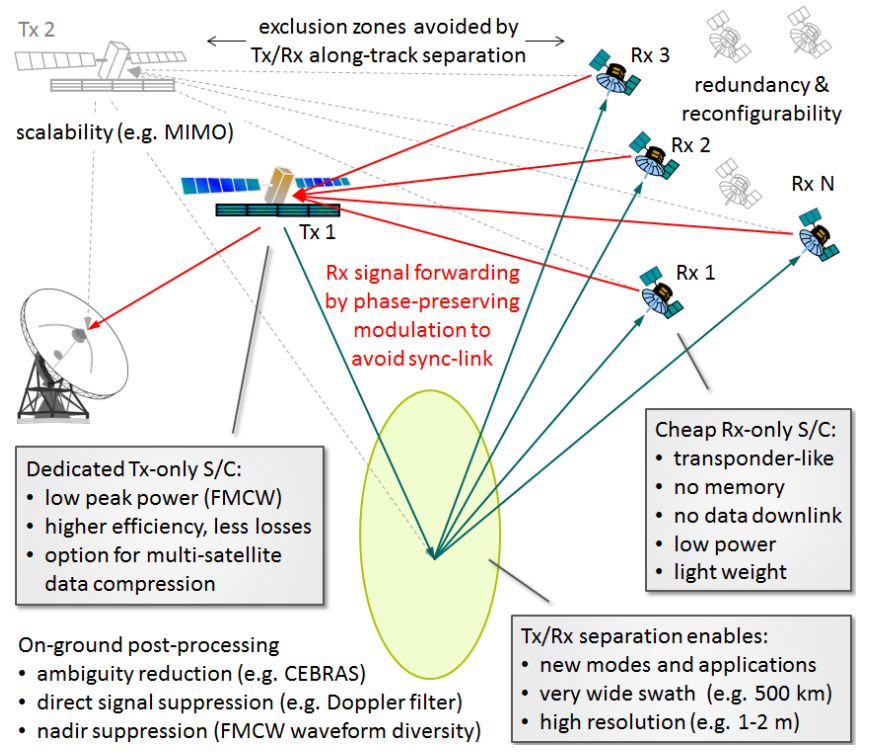

Figure 1: Illustration of the MirrorSAR concept. A wide scene is illuminated by one or multiple transmitter satellites. The scattered waves are spatially sampled by multiple receivers that route their radar echoes to the transmitter. The transmitter coherently demodulates and combines the forwarded signals, before the relevant information is transferred to the ground.

interferometry for traffic and ocean monitoring, polarimetric SAR interferometry for improved vegetation characterization, super-resolution and image enhancement in range and azimuth or even coherence tomography via the combination of multiple single-pass cross-track interferograms with varying baselines [3], [4]. Additional capabilities arise from multistatic SAR systems where a set of spatially distributed receivers acquire the scattered radar echoes from multiple directions [5]. This enhanced observation space opens the door towards multi-angular back- and forward scattering measurements for speckle reduction and improved scene characterization, multibaseline cross-track interferometry for the acquisition of ultra-high resolution DEMs without layover and phase ambiguities, or even single-pass SAR tomography for the observation of internal structure dynamics of semitransparent scatterers like vegetation and ice. Further opportunities are 3-D velocity measurements of moving objects and advanced vector deformation monitoring, the retrieval of tropospheric water vapor and ionospheric delays in case of high turbulence, as well as the retrieval of the ocean surface topography and its short- and long-term evolution over time. 
Despite these widespread opportunities, only one bistatic SAR system has yet been deployed in space. The main reason for this imbalance between opportunities and investment are the complexities and costs associated with biand multistatic SAR systems. Important cost drivers are the duplication of hardware for the reception, storage and downlink of radar data, the increased launch volume and mass for the deployment of multiple satellites, the necessity of additional hardware for accurate phase synchronization and the need of a high downlink capacity to transfer the radar data from multiple satellites to the ground. To tackle these challenges, we present in the following several new ideas and concepts that are not only suitable to reduce the hardware and downlink requirements for future bi- and multistatic SAR systems, but that may also provide a new approach to overcome the limits of conventional monostatic SAR systems in terms of swath width and spatial resolution.

\section{THE MIRRORSAR CONCEPT}

The MirrorSAR system architecture discussed in this paper comprises a set of spatially separated transmit and receive satellites that may be equipped with reflector antennas and/or direct radiating arrays. The system can be operated in various modes like a single-transmit single-receive (SISO), a single-transmit multiple-receive (SIMO) or even a multipletransmit multiple-receive (MIMO) mode as illustrated in Figure 1. A distinguishing feature of MirrorSAR is the use of a fractionated SAR approach where the hardware of the high-power radar transmitter and the hardware of the lowpower radar echo receiver are mutually separated by accommodating them on different platforms. This separation is not only well suited to independently optimize the transmit and receive chains and their sub-systems by, e.g., avoiding losses from $\mathrm{Tx} / \mathrm{Rx}$ switches and reducing thermal drifts and noise in the receiver chain, but it enables also new SAR imaging modes that rely on the continuous reception of radar echoes (cf. Section 3). The separation of the Tx and $\mathrm{Rx}$ satellites opens moreover the door for using longer transmit pulses or even a frequency modulated continuous wave (FMCW) illumination, which reduces the peak power requirements and bears therefore high potential to simplify the high power amplifier and its associated power supply system within the Tx satellite.

A further distinguishing feature of MirrorSAR is the radical simplification of the receiver satellites. This is achieved by reducing their main functionality to a transponder-like routing of the radar signals from the passive receivers to the transmitter(s), as illustrated in the upper right of Figure 2. As the receiver satellites act only as space relays, they do no longer need expensive data storage and downlink systems. This will in turn reduce their mass, power, accommodation and control demands, supporting the use of a small low-cost satellite bus.

Another key benefit of MirrorSAR is the opportunity to overcome the necessity of a bidirectional phase synchronization link between the transmitter and receiver satellites

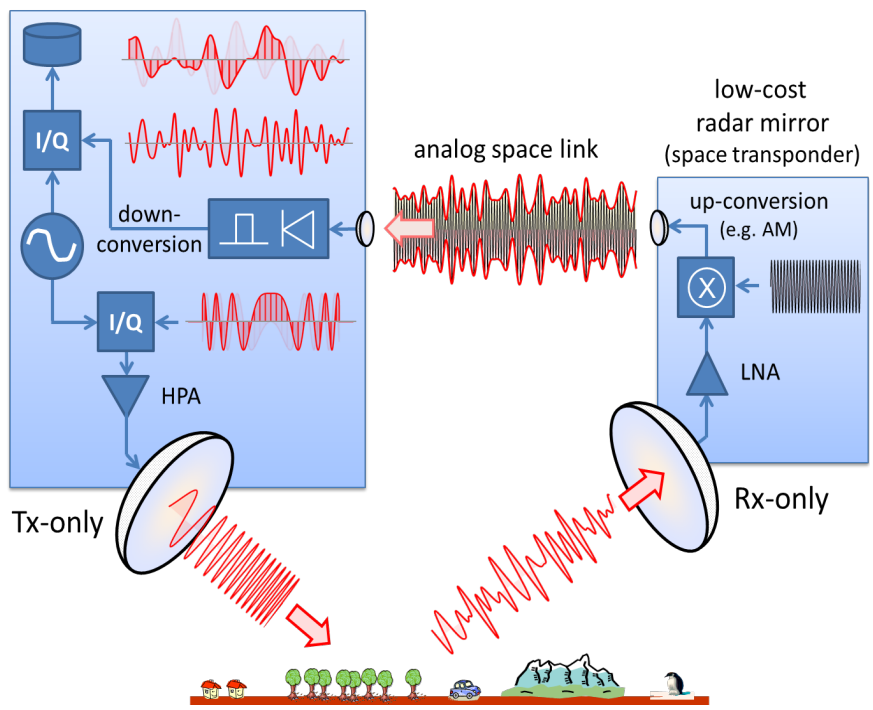

Figure 2: MirrorSAR employs a fractionated SAR approach where the high-power radar transmitter and the low-power radar echo receiver are accommodated on different platforms. The $R x$ satellite is considerably simplified by reducing its main functionality to a transponder-like routing of the radar signals. To preserve the signal integrity of the radar echo an appropriate modulation is employed (here: amplitude modulation).

without sacrificing bistatic and interferometric performance. For this, we require that the signal routing in the receiver preserves the phase of the radar signal up to a constant internal delay. In its most simple configuration, this might be achieved by limiting the receiver functionality to a mere amplification and re-radiation of the received RF signal. This option has, however, the drawback that the forwarded signal may interfere with the radar signal transmitted to the ground and its associated echo. A better solution, which moreover enables the forwarding of multiple channels from each receiver as well as from multiple satellites, is the use of a modulation that preserves the phase of the routed radar signal and avoids any dependency on the (possibly random) phase of the modulation carrier. This can be achieved by a variety of modulation techniques and as a simple example we may conceive that the receiver satellite generates for this purpose a high frequency signal (it might even be an optical carrier) that is amplitude modulated by the radar echo. As illustrated in Figure 2, an incoherent demodulation in the transmitter will then recover the time-delayed radar echo without phase disturbance from the high frequency carrier. After this first-stage down-conversion, the RF radar signal is, in a second step, demodulated to baseband by using, e.g., a coherent I/Q demodulator that is driven by the local oscillator in the transmitter. As this oscillator was also used to generate the transmitted radar pulse, possible frequency and phase drifts are cancelled as in a classical monostatic SAR. Radar echoes from multiple channels within each receiver, as well as from multiple receiver satellites, can then be routed without mutual interference by employing multiple carrier frequencies for the forwarded signals (imagine e.g. different "colors" in case of an optical carrier). 
An implicit assumption of this approach is that the distances between the transmitter and receiver satellites do not change abruptly and can be estimated with sufficient accuracy. The first assumption is justified by Newton's first law, while the required accuracy in the second assumption is to a large degree dictated by the specific application. If only bistatic SAR amplitude images are desired, coarse estimates of the relative position and velocity are sufficient. Single-pass interferometry and tomography will, on the other hand, ask for accurate relative phases and therefore precise position estimates between the involved satellites. Such accurate position estimates are, however, anyway required to avoid phase offsets of comparable magnitude that would otherwise arise from baseline errors. Experience with TanDEM-X shows that a relative position estimation with an accuracy in the order of $1 \mathrm{~mm}$ can be achieved based on conventional GPS technology [3]. This accuracy would already limit the residual phase errors to approx. $1.5^{\circ}, 6^{\circ}$ and $12^{\circ}$ in L-, Cand X-band, respectively. Note that these estimates refer to the total slowly varying phase offset and the much more relevant short-time relative phase variations will be by at least one order of magnitude smaller than these values.

MirrorSAR offers a further distinguishing advantage if employed in a multistatic SAR with multiple receiver satellites. For this, we first recall that a "conventional" approach with fully equipped receiver satellites requires the independent downlink of each Rx signal, which leads to a downlink volume that raises proportional to the number of receiver satellites. In MirrorSAR, the routing of the receiver signals to a centralized node opens, on the other hand, the door for an efficient on-board data compression, as the signals from the multiple receivers are, especially in case of close formations, typically characterized by a high degree of mutual redundancy. It is therefore possible to reduce the recorded information before downlink to a single master signal and a number of difference signals that are characterized by a significantly reduced entropy. Further opportunities for a considerable data reduction arise for interferometric applications like single- or multi-baseline DEM generation which do not need full resolution singlelook complex images, but only multi-looked interferograms.

\section{HIGH-RESOLUTION WIDE-SWATH IMAGING}

The spatial separation of the transmitter and receiver satellites enables also new modes for high-resolution wideswath SAR imaging [6]. For this, we consider the example in Figure 3, where the transmitter satellite on the upper left illuminates an ultra-wide swath via a broad antenna beam. In contrast to a monostatic system, there are no restrictions on the transmit duty cycle and one can even radiate a continuous, periodically frequency modulated chirped waveform. The scattered radar echoes are then recorded by the receiver satellite(s), one of which is shown on the upper right of Figure 3, carrying in this example a reflector antenna with multiple feeds. If we consider now the case of a SAR with high azimuth resolution, which requires in stripmap

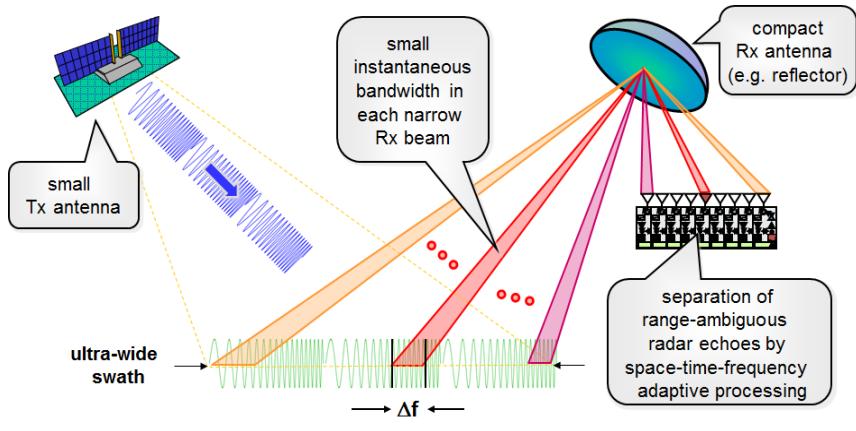

Figure 3: Bistatic SAR for high-resolution ultra-wide-swath imaging. The transmitter satellite illuminates a wide swath and the scattered radar echoes are recorded with multiple elevation beams by employing a multi-channel receiver.

mode a short antenna, the PRF has to be chosen sufficiently high to unambiguously sample the wide Doppler spectrum. Hence, the radar echoes from multiple transmit pulses will arrive at the same time at the receiver, which is a consequence of the wide swath illumination. Their direction of arrival will, however, differ due to the side-looking imaging geometry of the radar. It is therefore possible to separate these echoes by digital beamforming in elevation. This is illustrated in Figure 3 for the case of a reflector antenna where the echoes from the different Tx pulses excite different subsets of the feed array, and are therefore easy to separate from each other. For the case of very long transmit pulses, or in the limiting case of an uninterrupted FMCW illumination, this separation is, however, complicated by the fact that each Rx beam may only cover a portion of each transmitted pulse and that, at least for some swath positions, the echoes from two pulses may overlap within a single Rx beam. This challenge can, however, be easily resolved in the frequency domain by employing a frequency-dispersive beamforming which can be implemented in real-time by a short FIR filter [7]. The main advantage of the proposed bistatic SAR system concept is the capability to map an almost arbitrarily wide swath with high azimuth resolution in a stripmap-like mode.

To get a first idea about the capability of such a system and the required antenna sizes, we may consider an X-band SAR that shall map a $500 \mathrm{~km}$ wide swath with $1.5 \mathrm{~m}$ azimuth resolution in a single- or dual-polarized mode. The azimuth resolution of $1.5 \mathrm{~m}$ requires a Doppler spectrum of approx. $5 \mathrm{kHz}$ which can be illuminated in stripmap mode by a $3 \mathrm{~m}$ long antenna. In this first approximation, a PRF in the order of $7 \mathrm{kHz}$ is considered to provide a sufficient azimuth ambiguity-to-signal ratio. Assuming an orbit height of $800 \mathrm{~km}$ and a maximum incident angle of $50^{\circ}$, we can estimate the minimum required antenna height for the receiver satellite as $2 \mathrm{~m}$. In this approximation, we implicitly assume the use of a coherent range ambiguity suppression technique like CEBRAS [8]; otherwise a higher Rx antenna of 3-4 m would be required. It seems hence possible to implement an extremely powerful high-resolution ultra-wide swath system with unprecedented mapping capabilities by 
combining a compact Tx antenna with a length of $3 \mathrm{~m}$ and a height of less than $1 \mathrm{~m}$ with, for example, a passive receiver satellite that carries a reflector antenna with a diameter of 3$4 \mathrm{~m}$. Such a bistatic system would exceed the mapping capability of TerraSAR-X or TanDEM-X in terms of acquired resolution cells per second acquisition time by a factor of $(500 / 30) \cdot(3 / 1.5)=33.3$ and those of Sentinel-1 by a factor of $(500 / 240) \cdot(20 / 1.5)=27.8$, where we implicitly assume an unaltered range resolution.

One may ask whether the signal-routing in the MirrorSAR concept is applicable to such a multichannel SAR system, as the beamforming requires a coherent combination of multiple Rx channels. A possible solution to this problem is illustrated in Figure 4. The key idea is to divide the multichannel transponder-like receiver into two parts, where the first performs a coherent beamforming in the RF domain and the second modulates the beamformer outputs on different carriers such that they can be recovered in the transmitter without ambiguities (e.g. via a multifrequency amplitude modulation as illustrated in Figure 4). As an alternative to performing the beamforming directly in the RF domain, one may, as illustrated in the lower part of Figure 4, first demodulate the radar signals to baseband and then re-modulate the beamformer's output signals again to the RF band. This de-modulation/re-modulation approach is well suited to cancel phase errors that would otherwise be introduced by the local oscillator of the receiver satellite.

\section{CONCLUSIONS}

In this paper, we have introduced a novel SAR concept that employs a fractionated radar architecture to simplify the design of bistatic and multistatic SAR systems and missions. The main benefits are (1) the considerable simplification of the hardware and thermal design of the receiver satellites which lowers their mass, size and power demands, (2) the reduction of system losses due to the lack of circulators and switches, (3) the decreased peak power demands which reduce the complexity of the high-power amplifier and its associated power supply electronics in the transmitter satellite, (4) the opportunity for bistatic SAR synchronization without a dedicated synchronization link, and (5) the opportunity for efficient multi-satellite data compression which lowers the downlink requirements and reduces the mission operation costs. The bistatic multichannel system architecture discussed in this paper provides moreover an interesting alternative and in the future possibly even cheaper solution for the implementation of high-resolution ultra-wide swath SAR imaging systems, especially if multiple receiver satellites are required for the generation of advanced interferometric and/or tomographic products.

Additional opportunities arise by extending the proposed MirrorSAR architecture with multiple transmitters. This extension will not only increase the system redundancy, but it enables also new MIMO-SAR modes. Promising examples are a multiplicative increase of the interferometric baselines, new opportunities for multi-angular SAR imaging as well as

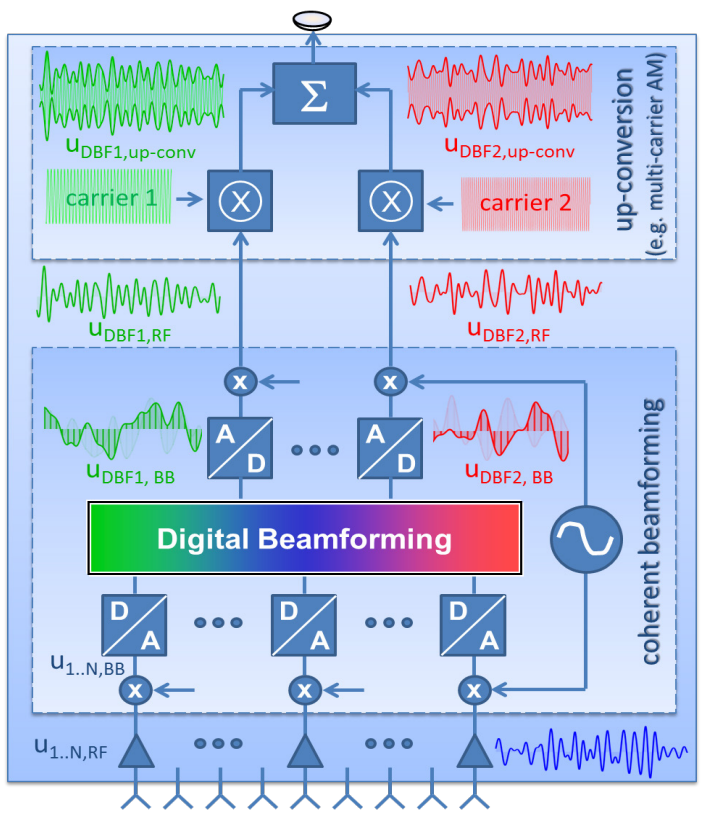

Figure 4: Multichannel MirrorSAR transponder with digital beamforming on receive. The integrity of the radar signal phase is preserved by performing first a coherent demodulation of the RF signals to baseband and a subsequent remodulation of the beamformer outputs to the RF band.

ionospheric and tropospheric measurements, and new MIMOSAR tomography modes [9]. Note that the separation between the transmitter and receiver, and the associated opportunity for simultaneous signal transmission and reception, allows for using much longer short-term shift-orthogonal Tx pulses. This will significantly alleviate the beamforming requirements for waveform separation within the individual receivers [10].

\section{REFERENCES}

[1] A. Moreira et al., "A tutorial on synthetic aperture radar," IEEE Geoscience and Remote Sensing Magazine, Vol. 1, No. 1, pp. 6-43, March 2013.

[2] A. Moccia et al., "Spaceborne bistatic synthetic aperture radar for remote sensing applications," International Journal of Remote Sensing, Vol. 21, No. 18, pp. 3395-3414, 2000.

[3] M. Zink et al., "TanDEM-X: the new global DEM takes shape," IEEE Geoscience and Remote Sens. Mag., Vol. 2, pp. 8-23, 2014.

[4] M. Rodriguez-Cassola et al., "First bistatic spaceborne SAR experiments with TanDEM-X," IEEE Geoscience and Remote Sensing Letters, Vol. 9, No. 1, pp. 33-37, 2012.

[5] G. Krieger and A. Moreira, "Multistatic SAR satellite formations: potentials and challenges," in Proc. IGARSS, Seoul, Korea, 2005.

[6] G. Krieger and A. Moreira, "Potentials of digital beamforming in biand multistatic SAR," in Proc. IGARSS, Toulouse, France, 2003.

[7] S. Huber et al., "Spaceborne reflector SAR systems with digital beamforming," IEEE Transactions on Aerospace and Electronic Systems, Vol. 48, No. 4, pp. 3473-3493, 2012.

[8] G. Krieger et al. "CEBRAS: Cross elevation beam range ambiguity suppression for high-resolution wide-swath and MIMO SAR imaging," in Proc. IGARSS, Milano, Italy, July 2015.

[9] G. Krieger et al., "MIMO-SAR tomography," in Proc. EUSAR, Hamburg, Germany, June 2016.

[10] G. Krieger, "MIMO-SAR: Opportunities and pitfalls," IEEE Trans. Geosci. and Remote Sensing, Vol. 52, No. 5, pp. 2628-2645, 2014. 